Hautarzt 2021 · 72:647-650

https://doi.org/10.1007/s00105-021-04863-9

Angenommen: 17. Juni 2021

(c) Springer Medizin Verlag $\mathrm{GmbH}$, ein Teil von Springer Nature 2021

\section{Hidradenitis suppurativa/Acne inversa: Von "orphan disease“ zu heilbarer entzündlicher Hauterkrankung}

\author{
Christos C. Zouboulis ${ }^{1,2}$ (D) $\cdot$ E. von Stebut ${ }^{3}$ \\ ${ }^{1}$ Hochschulklinik für Dermatologie, Venerologie und Allergologie, Immunologisches Zentrum, Städtisches \\ Klinikum Dessau, Medizinische Hochschule Brandenburg Theodor Fontane und Fakultät für \\ Gesundheitswissenschaften Brandenburg, Dessau, Deutschland \\ ${ }^{2}$ Hidradenitis Suppurativa Foundation e. V., Dessau, Deutschland \\ ${ }^{3}$ Klinik und Poliklinik für Dermatologie und Venerologie, Uniklinik Köln, Köln, Deutschland
}

Hidradenitis suppurativa/Acne inversa (HS) ist eine chronisch rezidivierende Hauterkrankung des terminalen Haarfollikels, die üblicherweise nach der Pubertät auftritt und vernarbend verlaufen kann. Sie manifestiert sich mit schmerzhaften, tief lokalisierten, entzündlichen Hautläsionen, die in apokrinen drüsenreichen Hautregionen auftreten, am häufigsten in den Axillen sowie der Inguinal- und Anogenitalregion (Dessauer Definition; $[9,26])$. Die aktuell berichtete weltweite Prävalenz beträgt 0,4\% [4], die Inzidenz in den USA liegt bei 6 Patienten pro 100.000 Einwohner und Jahr mit steigender Tendenz [21]. Es besteht eine starke Assoziation der Erkrankung mit aktivem Rauchen (relatives Risiko [RR] = 12,6; [17]) und erhöhtem Körpervolumenindex (BMl; $R R=1,1$ für jede BMI-Einheit, aber mit Prävalenz von 18,1 \% bei $\mathrm{BMI}>30$; $[7,17])$. Die primären diagnostischen Kriterien sind rezidivierende schmerzhafte oder eitrige Läsionen mehr als 2-mal in 6 Monaten in der Anamnese und die Beteiligung von Achselhöhlen, Leisten, Damm, Gesäßbereich und dem submammären Bereich bei der Frau [26]. Es treten Knötchen, Abszesse, Fistelgänge und Narbenbildung auf. Für die HS wurden bisher keine sicheren biologischen diagnostischen Marker nachgewiesen [5, 27].

HS ist eine entzündliche Hauterkrankung voller Widersprüche. Bis 2005 war sie eine Orphan-Erkrankung, seitdem wurde sie auf Initiative einer kleinen Gruppe von interessierten Dermatologen dem
Fach Dermatologie und Venerologie zugeteilt $[9,23]$. Kamen zur legendären ersten Dessauer Internationalen Konferenz nur 33 Teilnehmer, so waren es in 2021 schon 688 - aus 52 Ländern [23].

Initial als seltene Erkrankung betrachtet [9] wird sie heute als potenziell schwere Hautkrankheit klassifiziert und weist eine Reihe systemischer Komorbiditäten auf [3, 19, 25]. Während Diabetes Typ 2 und metabolisches Syndrom wichtige Begleiterkrankungen der HS in Europa und den USA mit einstelliger Prävalenz darstellen [12], sind in arabischen Ländern und Fernost $25 \%$ der HS-Patienten an Diabetes Typ 2 erkrankt [1]. Ein hochmalignes Plattenepithelkarzinom kann beim chronischen Befall der Anogenitalregion, aber nicht der Axillen auftreten [26].

Neben der klassischen sporadischen HS gibt es zahlreiche seltene HS-Varianten, wie die familiäre und die pädiatrische Formen sowie die syndromale HS [25]. Im Gegensatzzu den erwachsenen HS-Patienten rauchen HS-befallene Kinder nicht [20]. Es gibt Berichte mit großer Variation der HS-Prävalenz in verschiedenen Ländern, nämlich von $0,00033-4,1 \%$, die auch vom untersuchten Kollektiv und der angewandten Methode abhängig sind [26]. Während Frauen in der westlichen Welt am häufigsten betroffen sind [26], ist die absolute Mehrheit von HS-Patienten in Asien männlichen Geschlechts [1]. Während Afroamerikaner die höchste HS-Prävalenz in den USA aufweisen, sind HS-Daten aus Afri- 
ka kaum veröffentlicht [28]. Während eine bakterielle Kolonisation bei mildem Befall nicht nachgewiesen wurde [14], spricht die milde Form der HS auf lokales Clindamycin $1 \%$ am besten an [24]. Letztendlich bleibt eine auf pathophysiologischer Basis entwickelte Therapie noch ungewiss, da zurzeit weder die experimentelle Forschung noch die klinische Phänotypisierung [11] noch das breite Spektrum an nachgewiesenen Zielproteinen [27] sichere Ansätze bieten. Interessanterweise konnten auch "drug repurposing studies" den Kreis von guten Kandidaten für die HS-Therapie nicht weiter konkretisieren [29, 30].

HS bleibt daher die Hauterkrankung mit der deutlichsten Abnahme der Lebensqualität und der Arbeitsfähigkeit [10, 18], und ihre Therapie ist - trotz der häufigen Ineffektivität - mit hohen Kosten assoziiert [18]. Aus allen diesen Gründen ist kein Zufall, dass das Interesse der Ärzteschaft - und vor allen der Dermatologen, die die Betreuung von Patienten mit dieser Erkrankung koordinieren - wächst. Ergebnis dieses Interesses ist dieses Heft, zu welchem die Mehrheit der führenden HSSpezialisten, aber auch Patientenvertreter aus dem deutschsprachigen Raum beigetragen haben.

In ihrem Artikel „Epidemiologie, Patientenlebensqualität und Behandlungskosten der Hidradenitis suppurativa/Acne inversa" bestätigen Kirsten et al., dass die Prävalenz der HS wahrscheinlich unterschätzt ist, die Versorgung der HS mit hohen Kosten für das Gesundheitssystem und die Betroffenen einhergeht, die HS sich stark negativ auf die Lebensqualität (QoL) der Patienten auswirkt und die QoL beeinflussenden Faktoren multidimensional sind. Sie schlagen einen multidisziplinären Ansatz für das Risikomanagement dieser Krankheitspopulation vor [6].

In ihrem Beitrag weisen Nikolakis et al. auf die multifaktorielle Pathogenese der Erkrankung hin, wobei Rauchen und Übergewicht mit einer geringeren Wirksamkeit der systemischen Antibiotikatherapien assoziiert sind [13]. Das geringere Ausmaß der Verbesserung unter der Therapie mit Biologika im Vergleich zu den Ergebnissen bei anderen dermatologischen Erkrankungen spiegelt die Komplexität der pathophysiologischen Mechanismen der HS wider. Dies spricht für multimodale the- rapeutische Strategien, die auch von der genaueren Erkenntnis der Pathophysiologie der Erkrankung abhängt.

Von Laffert et al. berichten über die vielversprechenden neuesten Resultate der HS-Forschung und geben einen Einblick in das komplexe Krankheitsbild mit vielen potenziellen therapeutischen Zielen [22]. Daher sind sie optimistisch, dass mit einer Fülle von neuen Therapeutika zu rechnen ist, welche deutlich effektiver als heute aktive HS-Läsionen behandeln werden, aber auch die Entwicklung der Residualpathologien und damit die Rezidivneigung reduzieren sollten.

In ihrem Beitrag berichten Nikolakis und von Stebut über die topische Therapie der HS, wobei eine Applikation von Clindamycin, Resorcinol und die intraläsionale Injektion von Kortikosteroiden wirksame Behandlungen der milden HS darstellen [15]. Es existieren zusätzlich effektive apparative Behandlungsoptionen für die milde HS, wie eine Kombination aus speziellen intensiv gepulsten Lichtspektren und Radiofrequenz, die auch aufgrund der guten Verträglichkeit schon in sehr frühen Stadien und postoperativ zur Vermeidung von Rezidiven eingesetzt werden kann.

Pinter et al. weisen darauf hin, dass bei mittelschwerer bis schwerer HS eine Kombination von Clindamycin und Rifampicin (jeweils $600 \mathrm{mg} / \mathrm{Tag}$ ) für maximal 12 Wochen die empfohlene First-line-Therapie ist [16]. Die Gruppe der Tetrazykline stellt mit ähnlicher Wirkstärke zu Clindamycin/ Rifampicin eine wirksame Alternative dar. Acitretin ist begrenzt wirksam. Adalimumab ist als Langzeittherapie zugelassen und zeigt beste Effekte bei Hurley-Typ-IIPatienten.

Über die chirurgische Behandlung der nicht entzündlichen Formen der HS nach Hurley sowie die Möglichkeiten der medikamentösen und chirurgischen Therapie mit dem Ziel die Erkrankung erheblich zu verbessern, wird von Cramer et al. berichtet [2]. Da es an einheitlichen und validierten Daten fehlt, ist es derzeit nicht möglich, eine allgemein gültige Empfehlung bezüglich der Wahl bestimmter Resektions- und Rekonstruktionstechniken abzugeben. Allerdings scheint im Rahmen der aktuellen Datenlage die Indikation zur chirurgischen Behandlung, abgestimmt auf die Bedürfnisse des Patienten und die Er- fahrung des Operateurs, entscheidend zu sein. Die chirurgische Behandlung muss im Rahmen eines Kombinationstherapiekonzepts stehen, in welchem stets auch die Indikation zur medikamentösen Therapie geprüft werden sollte.

Zouboulis und von Stebut weisen in ihrem Beitrag darauf hin, dass Realworld-Daten zum besserem Verständnis der Erkrankung unabdingbar sind [31]. Aus Krankenkasseninformationen lassen sich notwendige Daten zu Prävalenz, Altersverteilung und anderen demographischen Details entnehmen. Real-worldDaten sind weiterhin wichtig, um aktuelle Therapieempfehlungen zu überprüfen. Die erhobenen Daten zeigen, dass es sich bei Adalimumab um eine kosteneffiziente, wirksame Therapie handelt. Unterstützende Maßnahmen wie Patientenschulungen helfen bei der Therapieadhärenz und damit deren Wirksamkeit. Letztlich hat sich der Baseline International HS Severity Score System (IHS4) als prädiktiver Faktor für Rezidive einer langfristigen Adalimumab-Therapie erwiesen.

In ihrem Beitrag aus der Praxis berichten Kurzen et al., dass im täglichen Management von HS-Patienten der Aufbau eines Vertrauensverhältnisses und die gleichzeitige Bereitschaft einer langfristigen Betreuung die wichtigsten Voraussetzungen für die erfolgreiche Behandlung sind [8]. Triggerfaktoren müssen individuell bestimmt werden, um den Verlauf möglichst frühzeitig positiv zu beeinflussen. Durch gute Aufklärung soll der Patienten in die Lage versetzt werden, eigenständig auf die Herausforderungen durch neue Abszesse oder Entzündungsschübe in bestehenden Fisteln zu reagieren. Die konsequente Anwendung der geeigneten physikalischen Therapien, Lokal- und Systemtherapeutika soll dem Patienten eine verbesserte Teilhabe und Lebensqualität ermöglichen.

Letztlich betonen Just und Winkler als Patientenvertreter, dass Betroffene eine Akzeptanz der vielfältigen Auswirkungen im sozialen und medizinischen Bereich und eine fachübergreifende Behandlung brauchen [5]. Sie betrachten den weiteren Ausbau von Fachkliniken und Weiterbildung von Ärzten, die eine adäquate Behandlung durchführen können, für dringend notwendig, damit Patienten zeitnah behandelt und auch in der nä- 
Hier steht eine Anzeige.

黑 Springer 
heren Umgebung einen Ansprechpartner finden.

HS ist die neueste der schweren, entzündlichen Erkrankungen im Verantwortungsbereich des Arztes für Dermatologie und Venerologie. Innerhalb der letzten 15 Jahre, während welcher HS intensiv klinisch und experimentell erforscht wurde, wurden zahlreiche Kenntnisse bei einer rasanten Entwicklung diagnostischer, prognostischer und therapeutischer Möglichkeiten erworben [25]. Es liegt sicher noch ein langer Weg vor uns, um die Erkrankung zufriedenstellend für alle Patienten behandeln zu können, doch schon heute kann folgendes gesagt werden: HS ist eine entzündliche Hauterkrankung, die man durch eine korrekte und konsequente Kombination der existierenden therapeutischen Möglichkeiten heilen kann.

Korrespondenzadresse

Univ.-Prof. Dr. med. Prof. honoraire Dr. h.c. Christos C. Zouboulis

Hochschulklinik für Dermatologie, Venerologie und Allergologie, Immunologisches Zentrum, Städtisches Klinikum Dessau, Medizinische Hochschule Brandenburg Theodor Fontane und Fakultät für Gesundheitswissenschaften Brandenburg

Auenweg 38, 06847 Dessau, Deutschland christos.zouboulis@mhb-fontane.de

Danksagung. Die Hochschulklinik für Dermatologie, Venerologie und Allergologie, Immunologisches Zentrum des Städtischen Klinikums Dessau ist Gesundheitsdienstleister der Europäischen Referenznetzwerkes für seltene und komplexe Hautkrankheiten (ERN Skin - ALLOCATE Skin group). EvS erhält Förderung aus dem ERA PerMed EU Projekt „Biomolecular Analyses for Tailored Medicine in AcneiNversa (BATMAN)".

Interessenkonflikt. C.C. Zouboulis hat Berater- und Vortragshonorare von Idorsia, Incyte, Inflarx, Janssen, Novartis, Regeneron und UCB erhalten, die nicht mit diesem Manuskript in Verbindung stehen. Seine Abteilung erhielt Honorare von AbbVie, Inflarx, Novartis and UCB für seine Teilnahme an klinischen Studien. E. von Stebut hat Berater- und Vortragshonorare von Almirall, Infectopharm, Janssen, Leo Pharma und Novartis erhalten, die nicht mit diesem Manuskript in Verbindung stehen.

\section{Literatur}

1. Chandran NS, Lee JH, Kurokawa I (2021) Hidradenitis suppurativa in south-east asia and east asia. Exp Dermatol 30(suppl 1):23-26

2. Cramer $P$, Schneider-Burrus $S$, Kovács $M$, Scholl

L, Podda M, Bechara FG (2021) Hidradenitis suppurativa/ Acne inversa - operative Optionen, Rekonstruktionen und die Kombination mit medikamentösen Therapien - Ein Update. Hautarzt. https://doi.org/10.1007/s00105-021-04864-8

3. Fimmel S, Zouboulis CC (2010) Comorbidities of hidradenitis suppurativa (acne inversa). Dermatoendocrinol 2:9-16

4. Jfri A, Nassim D, O'Brien E, Gulliver W, Nikolakis G, Zouboulis CC (2021) Prevalence of hidradenitis suppurativa: a systematic review and metaregression study. JAMA Dermatol. https://doi.org/ 10.1001/jamadermatol.2021.1677

5. Just E, Winkler T (2021) Acne inversa: eine seltene Erkrankung mit weitreichenden Auswirkungen für die Betroffenen - Erkenntnisse und Wünsche von Patienten. Hautarzt. https://doi.org/10.1007/ s00105-021-04861-x

6. Kirsten N, Frings V, Nikolakis G et al (2021) Epidemiologie, Patientenlebensqualität und Behandlungskosten der Hidradenitis suppurativa/ Acne inversa. Hautarzt. https://doi.org/10.1007/ s00105-021-04851-z

7. Kromann CB, Ibler KS, Kristiansen VB, Jemec GBE (2014) The influence of body weight on the prevalence and severity of hidradenitis suppurativa. Acta Derm Venereol 94:553-557

8. Kurzen H, Fritz K, Altenburg A (2021) Hidradenitis suppurativa/Acne inversa in der Praxis. Hautarzt. https://doi.org/10.1007/s00105-021-04850-0

9. Kurzen H, Kurokawa I, Jemec GBE et al (2008) What causes hidradenitis suppurativa? Exp Dermatol 17:455-472

10. Matusiak $Ł$, Bieniek A, Szepietowski JC (2010) Hidradenitis suppurativa markedly decreases quality of life and professional activity. J Am Acad Dermatol 62:706-708

11. Nikolakis G, Kaleta KP, Vaiopoulos A et al (2020) Phenotypes and pathophysiology of syndromic hidradenitis suppurativa: different faces of the same disease? A systematic review. Dermatology. https://doi.org/10.1159/000509873

12. Nikolakis G, Karagiannidis I, Vaiopoulos AG, Becker M, Zouboulis CC (2020) Endokrine Mechanismen bei der Pathophysiologie der Hidradenitis suppurativa. Hautarzt 71:762-771

13. Nikolakis G, Kokolakis G, Kaleta K et al (2021) Pathogenese der Hidradenitis suppurativa/Acne inversa. Hautarzt 72. https://doi.org/10.1007/ s00105-021-04853-x

14. Nikolakis G, Liakou Al, Bonovas S et al (2017) Bacterial colonization in hidradenitis suppurativa/ acne inversa: a cross-sectional study of 50 patients and review of the literature. Acta Derm Venereol 97:493-498

15. Nikolakis G, von Stebut E (2021) Lokale und neue apparative Therapien der milden Hidradenitis suppurativa. Hautarzt 72. https://doi.org/10.1007/ s00105-021-04849-7

16. Pinter A, Mrowietz U, Volz T (2021) Systemische Therapie der mittelschweren/schweren Hidradenitis suppurativa. Hautarzt 72. https://doi.org/10. 1007/s00105-021-04844-y

17. Revuz JE, Canoui-Poitrine F, Wolkenstein P et al (2008) Prevalence and factors associated with hidradenitis suppurativa: results from two casecontrol studies. J Am Acad Dermatol 59:596-601

18. Tzellos T, Yang H, Mu F, Calimlim B, Signorovitch J (2019) Impact of hidradenitis suppurativa on work loss, indirect costs and income. Br J Dermatol 181:147-514

19. Tzellos T, Zouboulis CC (2020) Review of comorbidities of hidradenitis suppurativa: Implications for daily clinical practice. Dermatol Ther (Heidelb) 10:63-71 (Erratum:2020;10:523)
20. Vaiopoulos AG, Nikolakis G, Zouboulis CC (2020) Hidradenitis suppurativa in pediatric patients: a retrospective monocentric study in Germany and review of the literature. J Eur Acad Dermatol Venereol 34:2140-2146

21. Vazquez BG, Alikhan A, Weaver AL et al (2013) Incidence of hidradenitis suppurativa and associated factors: a population-based study of Olmsted County, Minnesota. JInvest Dermatol 133:97-103

22. von Laffert M, Hunger RE, Navarini AA, Zouboulis CC (2021) Klinische, pathologische und molekulare Biomarker der Hidradenitis suppurativa/Acne inversa. Hautarzt 72. https://doi.org/10.1007/ s00105-021-04848-8

23. Zouboulis CC (2021) 10th European Hidradenitis Suppurativa (EHSF) e.V. Conference: a jubilee scientific event. Exp Dermatol 30(suppl 1):5-7

24. Zouboulis CC, Bechara FG, Fritz K et al (2012) S1 - Leitlinie zur Therapie der Hidradenitis suppurativa/Acne inversa (ICD-10 Ziffer: L73.2). JDtsch Dermatol Ges 10(Suppl 5):S1-S31

25. Zouboulis CC, Benhadou F, Byrd A et al (2020) What causes hidradenitis suppurativa? 15 years after. ExpDermatol 29:1154-1170

26. Zouboulis CC, del Marmol V, Mrowietz U, Prens E, Tzellos T, Jemec GBE (2015) Hidradenitis suppurativa/acne inversa: criteria for diagnosis, severity assessment, classification and disease evaluation. Dermatology 231:184-190

27. Zouboulis CC, Frew JW, Giamarellos-Bourboulis EJ et al (2021) Target molecules for future hidradenitis suppurativa treatment. Exp Dermatol 30(suppl 1):8-17

28. Zouboulis CC, Goyal M, Byrd AS (2021) Hidradenitis suppurativa in skin of color. Exp Dermatol 30(suppl 1):27-30

29. Zouboulis CC, Nogueira da Costa A (2021) Drug repurposing through drug-gene interaction profiles for hidradenitis suppurativa/acne inversa treatment. J Eur Acad Dermatol Venereol 35:e251-e254

30. Zouboulis CC, Readhead B, Dudley JT (2021) An additional drug repurposing study for hidradenitis suppurativa/acne inversa. Br J Dermatol 184:748-750

31. Zouboulis CC, von Stebut E (2021) Bedarf von Real-World-klinischen Studien für die Hidradenitis suppurativa/Acne inversa. Hautarzt 72. https://doi. org/10.1007/s00105-021-04847-9 